\title{
Dynamic assessment and its application to children with speech and language learning difficulties
}

\author{
JAMES LAW ${ }^{1} \&$ BERNARD CAMILLERI ${ }^{2}$ \\ ${ }^{1}$ Queen Margaret University, Edinburgh, UK, and ${ }^{2}$ City University, London, UK
}

\begin{abstract}
Speech-language pathologists, psychologists and others who assess children's performance have long been frustrated by performance variables that affect how a child performs but do not reflect ability as such. The shy child, the child who has had very little experience of tests, the child whose first language is not the language of the test situation, the child whose attention is poor all come into this category. They may not perform well on a test but their score does not reflect their real ability. There is a need to explore alternative ways of assessing the child's potential rather than reporting the result anyway and choosing to ignore those performance variables or simply dropping the test result altogether and saying that it is not possible to score the child. Dynamic assessment provides such an alternative.
\end{abstract}

\section{What is dynamic assessment?}

A consistent feature of conventional standardized assessments (sometimes referred to as "static") is that the assessor's input is kept to a minimum as the aim is to measure independent performance. Giving feedback to the individual being assessed is viewed as a source of measurement error. By comparison the defining feature of dynamic assessment is that it involves some form of instructional interaction between the assessor and the individual being assessed. The purpose of dynamic assessment is to reveal learning potential rather than to measure performance. The notion that cognitive functioning needs to be evaluated in an interactive context can be traced directly back to the writings of Vygotsky and Feuerstein which challenged the traditional separation between cognition as "natural" and instruction as "cultural". Like any creative process, the development of the field of dynamic assessment has spawned a whole range of characteristics which are adopted by some and not by others. All of the authors in this collection contextualise their work and the reader is encouraged to compare the introductions and the "approaches" that have been taken. Jeltova, Birney, Fredine, Jarvin, Sternberg, and Grigorenko (2007) helpfully outline a whole range of different procedures (test-teach-retest, learning test, graduated prompting, testing-thelimits) all of which would be considered dynamic but which have had different proponents over the years and which function in different ways.

For many years there has been a difference between those who feel that is possible to measure whatever it is that is dynamic in dynamic assessment and those who feel that the process is to too personal, too individualised to quantify. This is perhaps one of the reasons why there is a relative lack of published data on the reliability and validity of dynamic assessments. In this collection of papers we have come down firmly on the empirical side of the fence. All the papers in the present collection have included detailed descriptions of their procedures and have also demonstrated how the measurement works and what sort of data can be derived from the process.

Historically exponents of dynamic assessment have concentrated on assessing aspects of intellectual functioning which would be "statically" assessed by performance IQ tests. Frequently language has played an important part as the medium for the instructional interaction in dynamic assessments. There has been much less emphasis on the dynamic assessment of language skills per se. The work to date of Olswang, Peña and colleagues have led the way in the field. This collection of papers has been brought together to help broaden out the application of dynamic assessment to different aspects of language skills. As the name of this journal suggests the target audience is speech and language pathologists. But the implications go wider and hopefully

Correspondence: James Law, Centre for Integrated Healthcare Research, Queen Margaret University, Edinburgh EH12 8TS, UK. Tel: +44 (0)131 3173475. Fax: +44(0)131317 3162. E-mail: jlaw@qmu.ac.uk 
will be shared with a much wider group of professional, teachers who work with children with special needs, particularly those with communication difficulties of one sort or another and psychologists who are using dynamic assessment anyway. Of course, it would also be appropriate to list parents as amongst those who could better understand their child's abilities using dynamic assessment. How often have we heard them say "I am sure he could do much better than that if you tested him at home". Dynamic assessment provides a means of separating out real potential from wishful thinking.

\section{Overview of the included papers}

The papers reported here cover a variety of different groups of children, those with phonological difficulties, with Down syndrome, with Autism, with receptive language delay. Perhaps the area where it is easiest to see the process is that of phonological assessment because we already know a great deal about the detail of the process by which children acquire new sounds. Glaspey and Stoel-Gammon (2007) outline the development of the Scaffolding Scale of Stimulability, a hierarchy of cues and environmental manipulations that can be used to support a child in the production of phonemes. Of particular interest is their observation that dynamic and static assessment provide different results at the same point in time and across time. They appear to be tapping different aspects of learning. Donaldson and Olswang (2007) highlight the value of dynamic assessment of children with Autism arguing that it is the control of the context in the assessment process that is key to the successful measurement of performance in this group of children. Camilleri and Law (2007) look at preschool children with and without language impairment and their ability to respond to prompts in a word learning task and find that the children vary considerably in the response to prompts, something which appears to function relatively independent of language level. Alony and Kozulin (2007) also focus on receptive language skills but with children with Down syndrome and report that even a minimal amount of mediation improves language performance. Peña, Resendiz and
Gillam (2007) compare the responsivity to stimulation of older language impaired and language normal children and find that it is the cognitive strategies of the children which are the strongest predictors of language impairment. And finally Landor, Lauchlan, Carrigan and Kennedy's (2007) paper reports not on dynamic assessment itself but on the way in which the results are fed back to the child via video. Clearly this is a critical issue as far as the child's language is concerned. If the practitioner is not aware of the level of the child's understanding it is unlikely that it will be possible to feedback appropriately on the child's performance and thus the mediation will fail not because the child cannot respond to mediation but because the level of the mediation was wrong. This is an important study because of the high face validity of the procedure. It involves directly the child's class teacher. In other words it goes beyond relying on the specialist in dynamic assessment to carry out the mediation.

\section{Conclusions}

Dynamic assessment has the potential to open up a whole series of debates which are critical in the field of speech and language therapy. Is it really something dynamic about the assessment that is the active ingredient in dynamic assessment or is it simply because the type of reciprocal style which is implicit in dynamic assessment is effectively a feature of good teaching and what we are really doing is breaking down the characteristics of good teaching? Is it useful to distinguish between static or dynamic assessment? Are they simply accessing two very different features of the child's abilities. What impact is the interpretation of the difference likely to have on clinical decision making? And finally the empirical focus in these studies raises the question of whether it is really ever possible to manualize dynamic assessment. These approaches presuppose a fairly formal approach to dynamic assessment but does this effectively diminish or even do away with everything that is dynamic about the process. If we have to script what we do is there not a danger that the end result starts to approximate the structure of a static assessment and the "dynamic" element is lost? 
Copyright of Advances in Speech Language Pathology is the property of Taylor \& Francis Ltd and its content may not be copied or emailed to multiple sites or posted to a listserv without the copyright holder's express written permission. However, users may print, download, or email articles for individual use. 